\title{
SUMAG-7
}

Presented at the Particle Accelerator Conference, San Francisco, CA

March 12-14, 1979

\section{EIGHT-TESLA SYNCHROTRON DIPOLES -- DESIGN ALTERNATIVES}

William S. Gilbert, Glen R. Lambertson, and Robert B. Meuser

March 1979

Prepared for the U. S. Department of Energy under Contract W-7405-ENG-48

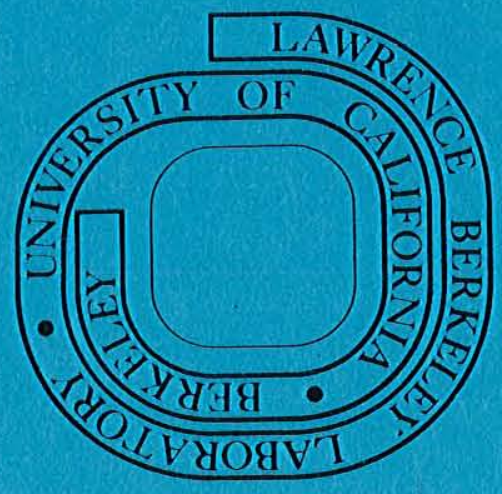




\section{LEGAL NOTICE}

This report was prepared as an account of work sponsored by the United States Government. Neither the United States nor the United States Department of Energy, nor any of their employees, nor any of their contractors, subcontractors, or their employees, makes any warranty, express or implied, or assumes any legal liability or responsibility for the accuracy, completeness or usefulness of any information, apparatus, product or process disclosed, or represents that its use would not infringe privately owned rights. 
William S. Gilbert, Glen R. Lambertson, and Robert B. Meuser**

\section{Abstract}

Superconducting accelerator bending magnets in the 4 to 5 tesla field range have been, or are being, developed for the LBL ESCAR, FNAL Doubler, and BNL ISABELLE projects and can be considered as state-of-the-art. We are now engaged in a program to develop accelerator dipoles with significantly enhanced performance, into the 8 -tesla range.

Analytical design studies, based on idealized configurations, have been done to trace the dependence of stresses within the coil and overall magnet size on the magnet aperture, coil current density, central magnetic field, structural ring stress, and magnetic induction in the iron flux-return yoke.

Our development program includes:

1. Development, and measurement at $4.2^{\circ} \mathrm{K}$, of coil structures consistent with the requirements of the analytical design studies.

2. Superfluid helium II at $1.8^{\circ} \mathrm{K}$ and at atmospheric pressure. Large magnets as well as conductors will be tested.

3. Development and evaluation of aluminum stabilization and various A-15 superconductors.

4. Tests of various model dipoles with instrumentation to measure mechanical motion.

\section{Analytical Design Studies}

A wide-range "parameter study" was undertaken, 1,2) using a mini-computer, to see how a variation of certain input. paraneters affected other output parameters. The model selected has a circular current winding with the current density uniform in radius and varying as $\cos \theta$, circular structural rings outside the winding, then the helium vessel and vacuum chamber, and outside of that, the warm-iron flux-return yoke, assumed to have infinite permeability.

In order to minimize the computational work involved, and the amount of output to be digested, the number of variables must be minimized. And for the same rea son, rather than investigate all possible combinations, one parameter at a time was varied while all other independent parameters were held constant at their "base values". Not all of the independent parameters affect all of the dependent parameters. The input parameters selected were: Coil inside radius; field in the aperture; field at the inside surface of the iron yoke; average flux density within the iron yoke legs; current density; and stress in the structural rings. The output parameters were: Coil outside radius; structural ring outside radius; iron yoke inside radius; iron yoke outside semi-width; maximum radial stress in the coil; and maximum tangential stress in the coil.

A selected portion of the parameter study is presented here. The results are shown in Table I and in Figures 1,2 , and 3 . Figure 1 shows our base-case, 8-tesla dipole, and Table 1 lists the various quantities of interest. Figure 2 shows how the overall magnet width and coil stress vary with central field, for a moderately high current density of $30 \mathrm{kA} / \mathrm{cm}^{2}$. Figure 3 shows the width and stress for a 8-tesla magnet as a function of coil current density.

*This work was supported by the Office of High Energy and Nuclear Physics Div. of the U.S. Dept. of Energy under contract N0. W-7405-ENG-48.

**Lawrence Berkeley Laboratory, Berkeley, CA 94720

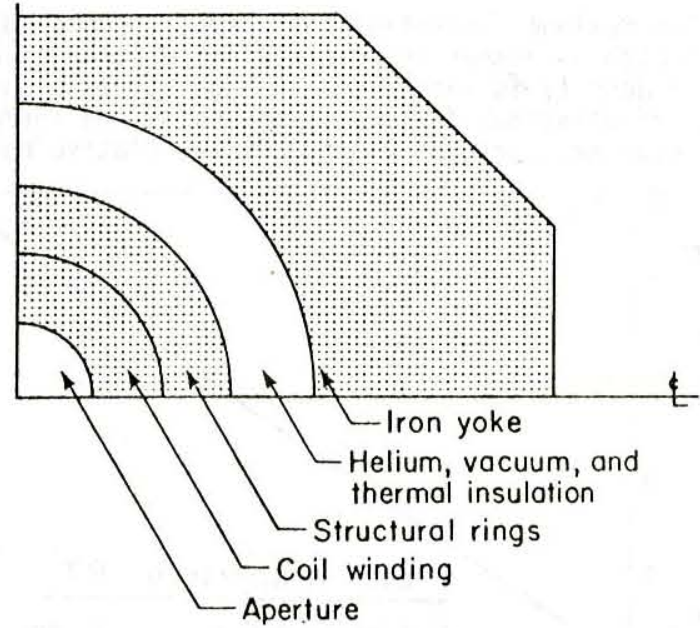

Fig. 1. Computer-generated quarter cross-section of base-case magnet for parameter study.

Table I.

Base Case for Parameter Study

Coil Inside Radius

Field in Aperture

Field at Iron Surface

Field in York Leg, Avg.

Current Density

Stress in Structural Ring

Coil Outside Radius

Structural Ring Outside Radius

Yoke Inside Radius

Yoke Outside Semi-Width

Radial Stress in Coil, Max.

Tangential Stress in Coil, Max.

Certain parameters are fixed at reasonably optimistic values. The coil inside radius of $4 \mathrm{~cm}$ is for PEP II, a proton ring in the PEP tunnel. The maximum field strength at the iron inside surface is 2 tesla. Previous studies have shown that much higher values can be used before central-field aberrations caused by saturation become a problem, and higher values result in a greater contribution of the iron to the central field. But higher values result in there being insufficient space between the structural rings and the warm iron for helium, vacuum, and thermal insultaion. The average flux density in the yoke legs is 2.5 tesla. This is low enough to avoid harmful effects of saturation on the central field, but might result in excessive stray flux, depending on the particular application.

The maximum stress in the structural rings is $50000 \mathrm{psi}$. This is low enough to avoid fatigue failure for cold-worked austenitic stainless steels and even for high-strength aluminum alloys in applications where a life of much less than $10^{6}$ cycles is tolerable. For determining the stresses within the coil and the forces transmitted to the structural-ring system, the coil was considered to be made up of infinitesimal keystone-shaped conductors, bounded by radial and constantradius lines, and free to slide on each other. Analytical formulas ${ }^{3)}$ give the stresses directly.

The structural-ring system was considered to cover the entire coil and to extend outward from the outer radius of the coil. Stresses were calculated from exact analytical formulas for a continuous uniform ring. 4 ) Although the stress was selected as the governing cri- 
terion for the sizing of the structural rings, the deflections might well be limiting. Ring distortion leads to aberrations in the central field and may lead to fatigue failure of the coil.

The maximum tangential coil stress becomes distressingly high as either the central field strength or the current density is increased. A high stress might lead directly to fatigue failure of the coil, and there might be a secondary sort of failure due to relative motion,

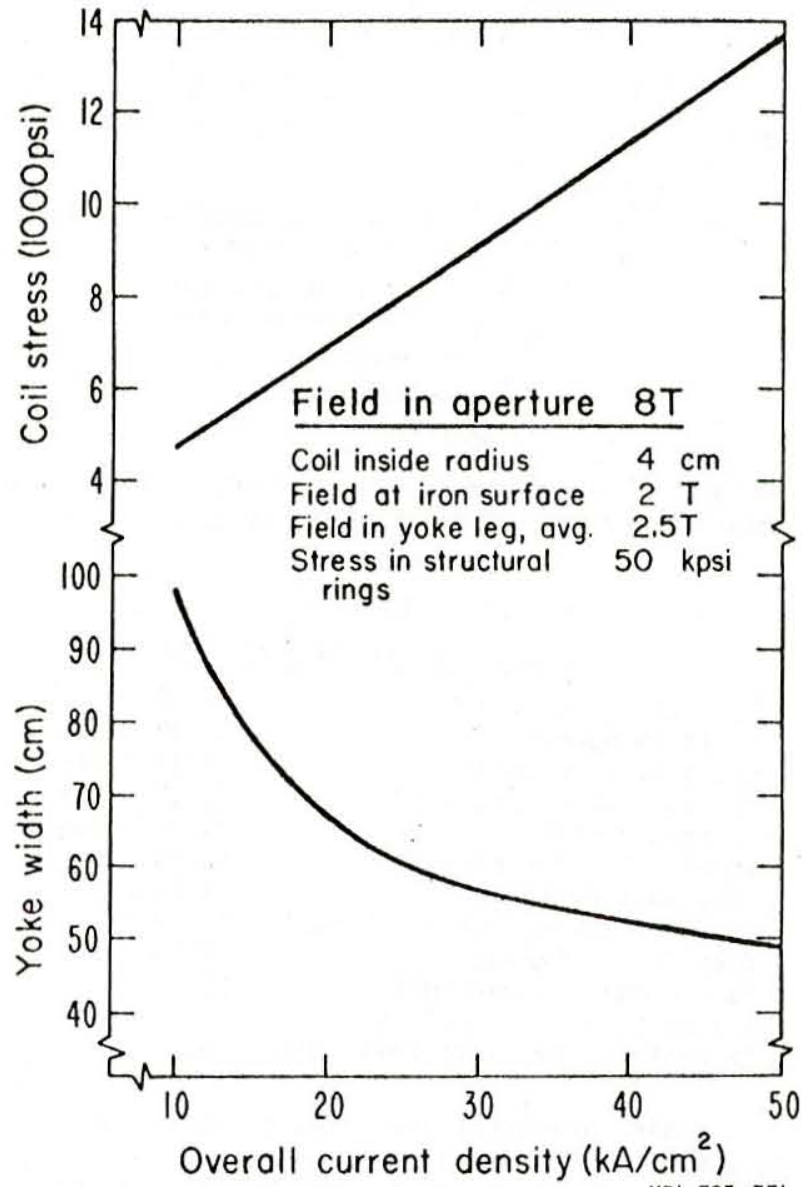

$X B L 793-771$

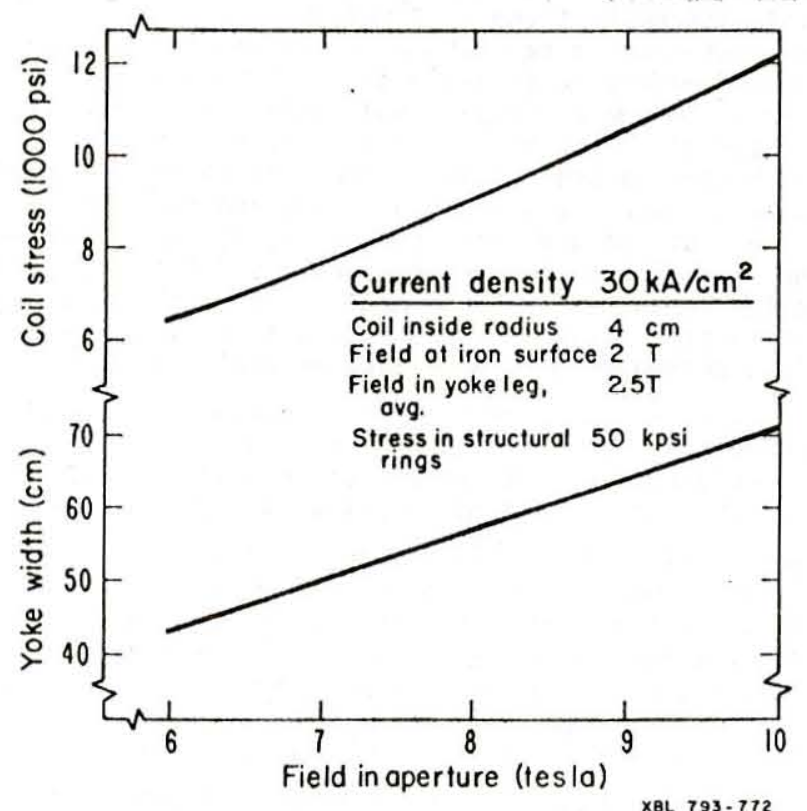

Fig. 2 and 3. The effects of magnetic field in the aperture and coil current density on the overall width and maximum coil compressive stresses. and attendant wear, between parts of the coil, and bet- 1 ween the coil and the structural rings. Large coil motion also results in premature quenching, training difficulties, and aberrations in the central field. A high elastic modulus reduces the motion, of course. But even achieving a suitably high modulus for the relatively mild requirements of the ESCAR and FNAL Doubler magnets was a difficult task.

\section{Coil Structure Development}

The mechanical behavior of the built-up coil structure under the Lorentz electromotive and restraintstructure forces have proved responsible for much of the degradation and training observed in high-current-density accelerator dipoles. The requirements for helium coolant passages and electrical insulation usually result in undesirably low compressive moduli. New materials and methods of coil fabrication are constantly being introduced. Mechanical testing of components and models of coil structure are usually done at room temperature.

We have instrumented a 5000-pound testing machine to perform mechanical tests down to $4^{\circ} \mathrm{K}$, and soon LBL.'s Materials and Molecular Research Division will be able to do similar testing at loads up to 50000 pounds.

\section{Superfluid Helium II, Pressurized at $1.8^{\circ} \mathrm{K}$}

We have under construction a 150-1iter helium II bath that will operate at $1.8^{\circ} \mathrm{K}$ and one atmosphere. Both superconductor current capacity and the thermal heat transfer are improved in superfluid helium at atmospheric pressure as compared to pool boiling normal helium at $4.2^{\circ} \mathrm{K}$. Remarkable increases have been observed in the superconducting stability of short samples. We plan to study the stability margins of sizable magnets -- 1-meter-long dipoles -- in the new facility.

The increase in the current capacity of NbTi at $1.8^{\circ} \mathrm{K}$ is displayed in Figures 4 and 5 , which also plot the current of $\mathrm{NbTi}$ at $4.2^{\circ} \mathrm{K}$ and multifilimentary $\mathrm{Nb}_{3} \mathrm{Sn}$ produced by the bronze process. The $\mathrm{Nb}_{3} \mathrm{Sn}$ currents are among the highest quoted in the literature ${ }^{5}$ but are still somevihat below the values achieved for the older, more developed, tape form of $\mathrm{Nb}_{3} \mathrm{Sn}$. Therefore, some increase in the current capability of multi-filamentary $\mathrm{Nb}_{3} \mathrm{Sn}$ might be in the offing. A copper to bronze + $\mathrm{Nb}_{3} \mathrm{Sn}$ ratio of $2:$; was assumed in generating the overall coil current-density numbers although the amount of stabilitation required for $\mathrm{Nb}_{3} \mathrm{Sn}$ is not well established.

The NbTi data are for the best high field alloy tested and reported in the literature. ${ }^{6)}$ Some data exist for $\mathrm{NbTi}$ current densities at $1.8^{\circ} \mathrm{K}$ and other estimates were generated from data supplied by Robert Schermer of LASL. ${ }^{7)}$ A rather low ratio of $\mathrm{Cu}$ to $\mathrm{NbTi}$ of $1: 1$ was assumed because at high field and $4.2^{\circ} \mathrm{K}$ the current capacity of the $\mathrm{NbTi}$ is quite low. For both the $\mathrm{Nb}_{3} \mathrm{Sn}$ and $\mathrm{NbTi}$, the coil space factor, the fraction of coil volume occupied by superconductor plus stabilizer, was taken to be a fairly high $2 / 3$. All the above assumptions are on the optimistic or high-current-density side, and one should probably plan on slightly lower current densities than appear on Figure 5.

The enhanced current capability of $\mathrm{NbTi}$ at $1.8^{\circ} \mathrm{K}$ compared with $\mathrm{Nb}_{3} \mathrm{Sn}$ at $4.2^{\circ} \mathrm{K}$ shows its promise up to at least 12 tesla. Even at $4.2^{\circ} \mathrm{K}$, NbTi coils seem competitive with $\mathrm{Nb}_{3} \mathrm{Sn}$ up to 8 tesla, but the temperature margin there might be inadequate. 


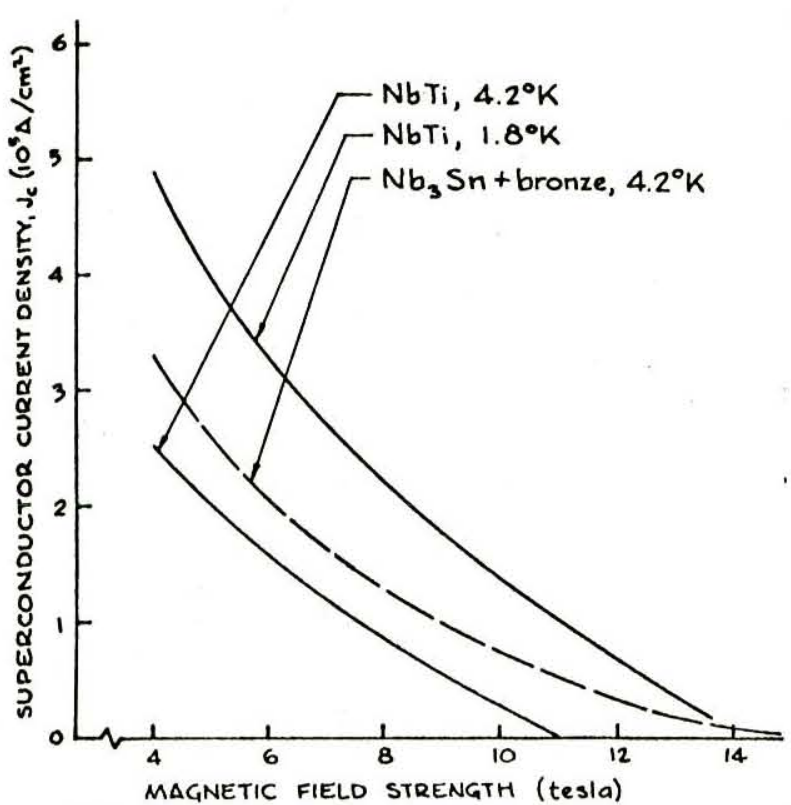

Fig. 4. Critical current density vs. magnetic field and temperature for various superconductors.

\section{Superconductor Development and Evaluation}

Aluminum Stabilized Conductor.

The use of ultra-pure aluminum (RRR $\geq 3000$ ) as a stabilizer in composite superconductor allows one, in principle, to have a cryostable conductor at a current density above $10 \mathrm{kA} / \mathrm{cm}^{2}$. This could eliminate training as a problem and facilitate the design and development of high-field magnets in a timely, predictable, and econonical manner. Samples are being fabricated for us, and evaluation experiments are planned.

$\mathrm{Nb}_{3}$ Sn Multi-Filament and Advanced Superconductors.

The advanced superconducting intermetallic compounds are brittle as compared with the NbTi now in general use. Their use requires new fabrication techniques, one of which is to wind the magnet with unreacted conductor, and then form the $\mathrm{Nb}_{3} \mathrm{Sn}$ in situ through a

high-temperature heat treatment. We have procured some unreacted multi-filament $\mathrm{Nb}_{3} \mathrm{Sn}$ and plan to work with the materials specialists in the LBL MMRD division on winding, heat treating, and insulation techniques.

$\mathrm{Nb}_{3} \mathrm{Al}, \mathrm{Nb}_{3} \mathrm{Ge}, \mathrm{Nb}_{3}(\mathrm{Al}, \mathrm{Ge})$ all have superior superconducting properties to those of $\mathrm{Nb}_{3} \mathrm{Sn}$ and all have been fabricated by the MMRD materials group group. Possible advantages of fabricating magnets from these materials will be explored.

\section{Design Alternatives}

The overall coil current densities, shown in Figure 5 , display the alternatives between temperature and materials that one has to work with. Figures 2 and 3 show how these current densities affect the magnet overall size and coil stress. The problems of various development stratfgies thus become fairly clear, although the solutions are far less so. In addition to the superconductor materials and operating temperature options mentioned above there are also choices of superconductor configurations, insulation, glues, and other materials of construction.

We have also given attention to alternative magnet configurations. Previously, we have built magnets having circular structural rings, to resist the bending resulting from non-uniform radial loads, only on the

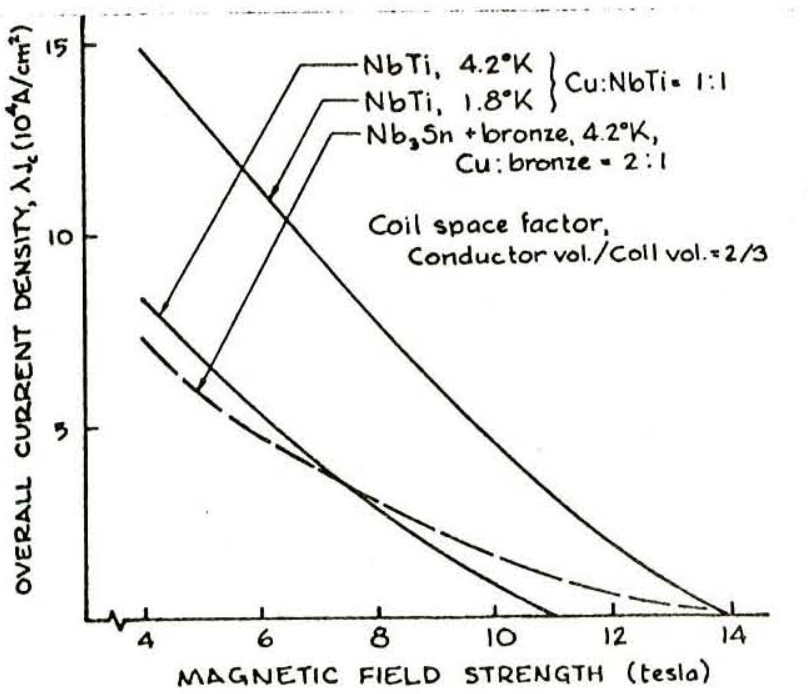

Fig. 5. Overall coil current density vs. magnetic field and temperature.

outside of the coil. We have also built magnets having all of the required bending resistance provided by a tube on the inside of the coil. This works well in cases where the coil thickness is relatively large. For high-field, small-aperture magnets putting all or part of the bending-resistant structure on the inside might be the preferable alternative. We have given some attention to coils with oval cross sections. In principle one should be able to design a coil in such a way that the banding is in pure tension, with no bending moments whatsoever. Attempts at LBL to design such magnets, several years ago, were not successful, but it is possible to reduce the bending to a small value by trial-and-error. Since the design of circular structural rings is dominated by bending, and the direct stress is small, reduction of the bending would certainly be desirable provided the fabrication problems can be solved. The general problem of increasing the efficiency of the structure applies not only to the relatively small-cross-section accelerator dipoles but even more to the large dipoles required in MHD. We are following the development of structural designs in the MHD program.

\section{References}

1. R. Meuser, Systems Analys is of Superconducting Bending Magnets -- A Mini-Computer Program, Lawrence Berkeley Laboratory Report LBID-004 (Eng. Note M-5309), March 1979.

2. R. Meuser, Superconducting Magnet Parameter Study, Lawrence Berkeley Laboratory Report LBID-005 (Eng. Note M-5310), March 1979.

3. R. Meuser, Stresses in a Thick $\operatorname{Cos} n \theta$ Winding, Lawrence Berkeley Laboratory Report UCID-8083 (Eng. Note M-5256), November 1978.

4. R. Meuser, Stresses in a Ring with a $\operatorname{Sin} n \theta$ or $\operatorname{Cos}$ $n \theta$ Distribution of Shear and Normal Surface Forces, Lawrence Berkeley Laboratory Report UCID-8076

(Eng. Note M-5255) October 1978.

5. C.H. Rosner; Cryogenics Worldwide-USA. Superconductivitity at Intermagnetics General; Cryogenics v. 19 n. 1, 3-9, Jan. 1979.

6. H.R. Segal, et al., Development of NbTi Superconductor for High Field Applications, IEEE Trans. on Magnetics, v. MAG-15 n. 1, 807-809, January 1979.

7. R. Schermer, Los Alamos Scientific Laboratory personal communication, 1979. 